\title{
Advances in stool DNA testing lead to improved colorectal cancer detection rates
}

Researchers from the USA have taken advantage of improvements to stool DNA testing to advance noninvasive screening for colorectal cancer (CRC). Their study, published in the New England Journal of Medicine, reports improved sensitivity of a multitarget stool DNA test compared with a standard faecal immunochemical test (FIT) in detecting CRC. "This is a marked improvement in cancer sensitivity over earlier versions of the test, making it the most sensitive noninvasive test for colorectal cancer to date," says Thomas Imperiale, lead author.

The method used in this study has more genetic markers and applies advances in technology for the analysis. These markers are for activating point mutations in KRAS and inactivating promoter methylation of BMP3 and NDRG4. Better sample preservation and a haemoglobin immunoassay further differentiate this method from earlier versions of the test.
The prospective, multicentre study included 9,899 asymptomatic people (aged 50-84 years) with an average risk of CRC. Prior to colonoscopy, participants provided stool samples, which were subject to DNA testing and a commercially available FIT. The colonoscopy findings were used to assess the sensitivity and accuracy of each test for CRC and detection of precancerous lesions.

Of the $65 \mathrm{CRC}$ and 757 precancerous lesions confirmed by colonoscopy, the DNA test identified 60 (92.3\%) cancers and 321 (42.4\%) precancerous lesions. These results were considerably higher than for FIT, which identified $48(73.8 \%)$ cancers and $180(23.8 \%)$ precancerous lesions.

Despite higher sensitivity, the DNA test was less specific than FIT $(89.8 \%$ versus $96.4 \%$ respectively) resulting in more false-positives. Nevertheless, Imperiale explains that although specificity of the stool DNA test was inferior to FIT, this decrease in specificity might be considered reasonable in light of the high sensitivity of the DNA test.

\section{1 ...the most sensitive noninvasive test for colorectal cancer to date... 77}

The development of a highly sensitive stool-based test could increase patient uptake and adherence to screening and help to decrease the incidence of CRC. However, test accuracy remains a substantial priority as improved reliability will reduce unnecessary follow-up tests and anxiety about cancer.

Gillian Patman

Original article Imperiale, T. F. et al. Multitarget stool DNA testing for colorectal-cancer screening. N. Engl. J. Med. doi:10.1056/NEJMoa1311194 